\title{
Sound source separation based on time reversal technique in room
}

\author{
Jinfang Zeng ${ }^{\mathrm{a}}$, Lintao $\mathrm{Xu}^{\mathrm{b}}$, Yicheng Zeng ${ }^{\mathrm{c}}$, Bing Bai ${ }^{\mathrm{d}}$ \\ School of Physics and Optoelectronics, Xiangtan University, Xiangtan 411105, China \\ a zengjinfang@xtu.edu.cn, b979229325@qq.com, cyichengz@xtu.edu.cn, d120465498@qq.com
}

Keywords: time reversal; source separation; microphone array; temporal compression

Abstract: Sound source separation (SSS) is a very important processing in room with multi-source. Common methods for SSS do not perform well in reverberation room. Time reversal technique (TRT) has many advantages to provide a solution for room acoustic problem. We propose a novel method of SSS based on TRT (TRS) in reverberation room. The presented method can separate specific sources from mixed signals and reduce reverberation and noise. The theory of TRS is derived and the numerical simulations are proposed.

\section{Introduction}

In room acoustic environment, such as meeting room, classroom, or lecture hall, often more than one sound source are simultaneously present. The signal reaching a mic is composed of various sources. Usually we are only interested in specific sources. Consequently, a processing of SSS with high quality is requested, which untangles mixtures into individual sources without other interfering sources, just as "Cocktail party effect". A processing of SSS can also be as a preprocessing stage of acoustic processing used in sound measurement and recognition, etc. The most challenge for SSS in reverberation room is the multi-path effect caused by rich reflection and reverberation. Several methods for SSS, e.g. DSB[1], Blind source separation (BSS)[2,3], Computational auditory scene analysis (CASA)[4], and Time-frequency masking (TFM) have been utilized in diverse fields successfully, but they do not perform well in reverberant environment. About to under-determined / over-determined problem, instantaneous / convolution mixture, and noise, they do not provide overall solutions[5]. Moreover, these methods' performances will decrease with increased complexity of environment. TRT is a well-known technique based on acoustic reciprocity theorem and time reversal invariance[6]. The main properties of TRT are as follows: (1) TRT can achieve temporal compression and spatial focusing; (2) TRT can obtain signal focusing without any prior knowledge of transducer array and medium properties[7]; (3) TRT can use the multi-path effect[8]; (4) M.Fink indicated "time reversal is a very robust technique compared to time-delay focusing techniques"[9,10]; (5) TRT is applicable to under-determined problem. TRT has been applied in ultrasound and electromagnetic ranges. Recently, some works have been done in audible range, such as source location in room[10] and outdoor[11]. However, TRT's application on SSS has been seldom discussed.

In the present paper, TRS will be proposed in view of TRT's advantages, which can separate multi-source from mixed signals to focus interested sources and reduce reverberation, reflection, and noise in reverberation room.

\section{TRS method}

Supposing that there are $\mathrm{m}$ sources and $\mathrm{r}$ mics in sound field where $S_{m}$ and $M_{r}$ denote the m-th 
source and r-th mic respectively; $s_{m}(t)$ and $y_{r}(t)$ are the source signal and received signal corresponding with $S_{m}$ and $M_{r} . h_{m r}$ and $h_{m r}^{\prime}$ denote the equivalent channel impulse responses (CIR) of the forward (from the m-th source to the r-th mic) and backward (from the $r$-th mic to the m-th source) channels respectively. Assuming that the backward channel matches with the forward channel, i.e. $h_{m r}=h_{m r}^{\prime}$, according to the basic theory of TRT, the output of TRS (the m-th separation signal) and corresponding spectrum are derived as following:

$$
\begin{aligned}
& S_{m}^{t r}(t)=S_{m}(t) \otimes \sum_{i=1}^{r} R_{h_{m i}}(t) \\
& S_{m}^{t r}(\omega)=S_{m}(\omega) \cdot \sum_{i=1}^{r}\left|H_{m i}(\omega)\right|^{2}
\end{aligned}
$$

Where $\sum_{i=1}^{r} R_{h_{m i}}(t)$ and $\sum_{i=1}^{r}\left|H_{m i}(\omega)\right|^{2}$ are the sum of autocorrelations and the sum of energy spectrums of CIRs between the m-th source and all mics respectively. $\sum_{i=1}^{r} R_{h_{m i}}(t)$ and $\sum_{i=1}^{r}\left|H_{m i}(\omega)\right|^{2}$ are the equivalent CIR and transfer function of TRS for the m-th source. Eq. (1) and (2) tell us that the separation signal is convolving the original signal with a Dirac delta function, which is similar to the original signal and its spectrum is $\sum_{i=1}^{r}\left|H_{m i}(\omega)\right|^{2}$ times of that of the original signal. We can come to a conclusion that a separation signal at a given source location involves only its own source component without other sources' components. Thus multi-source is separated from mixed array signals. Also, TRS just changes a signal's amplitude and does not change its frequency and phase.

\section{Numerical Simulations}

To verify the proposed algorithm, we carry out some simulations in MATLAB. The room acoustic model is build based on "Audio systems array processing toolbox(ASAPT)". The image method[12] is used to simulate the reverberation room. Based on ASAPT, we program the algorithm of TRT and simulate TRS. The simulations mainly focus on the separation performances of sources in a 3D rectangular reverberation room with the size of 3.6Õ 3.6Õ $2.2 \mathrm{~m}$. There are six sources(S1-S6) and sixteen mics(M1-M16) in the room model. The sources and mic array are scattered. In simulations, the original signals emitting from S1-S6 are six speeches denoted by s1-s6, which have been utilized in ASAPT. Six speeches are all target sources. The array signals in M1-M16 are denoted by m1-m16; six separation signals of TRS are denoted by tr1-tr6. According to "Sabine reverberation formula", the reverberation time (RT) of room can be calculated by the room volume and absorption amount. So we vary RT of room through varying the reflection coefficient(RC) of room.

\section{Basic simulation}

Under the simulation condition of $\mathrm{RT}=116 \mathrm{~ms}$ and $\mathrm{SNR}=-5 \mathrm{~dB}$, we compare the separation performances of source with non-source. The correlation coefficients between each of tr1-tr6 and s1-s6 are illustated in Fig.1. 


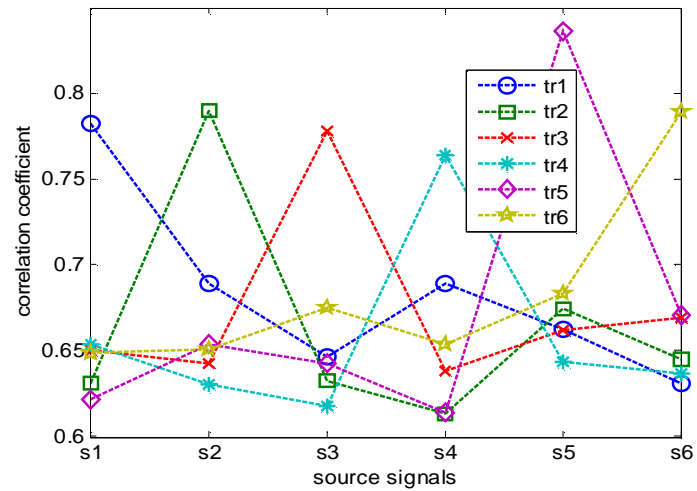

Fig.1 Correlation coefficients between tr1-tr6 and s1-s6 with RT $=116 \mathrm{~ms}$ and $\mathrm{SNR}=-5 \mathrm{~dB}$.

As seen from Fig.1, under reverberation and noise, the correlation coefficients of tr1-s1, tr2-s2, tr3-s3, tr4-s4, tr5-s5, and tr6-s6 are 0.7830, 0.7902, 0.7781, 0.7644, 0.8361, and 0.7891 respectively. The similarities are bigger for sources than non-source. Six sources are separated successfully and TRS has good separation effect.

\section{Effect of reverberation}

This section discusses the effect of reverberation on separation by selecting different RT. Under basic simulation condition and $\mathrm{SNR}=-15 \mathrm{~dB}$, the correlation coefficients between the separation signals and original signals for TRS and DSB are illustrated in Fig.2.
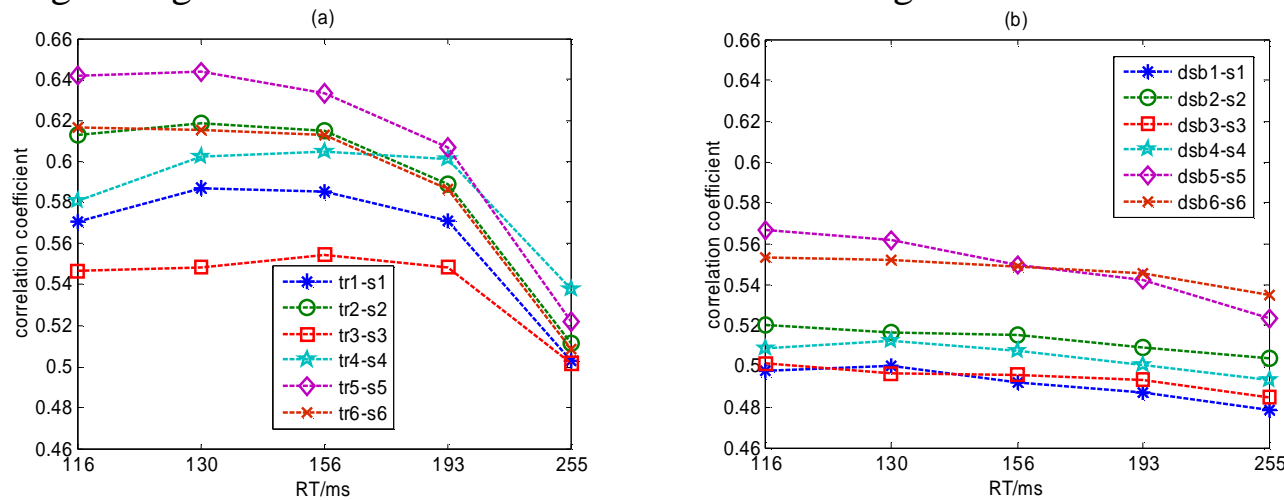

Fig.2 (a) Correlation coefficients between tr1-tr6 and s1-s6 with different RT and SNR $=-15 \mathrm{~dB}$. (b) Correlation coefficients between dsb1-dsb6 and s1-s6 with different RT and SNR $=-15 \mathrm{~dB}$.

As seen from Fig.2 (a), for TRS, the correlation coefficients for most of sources decrease with RT increasing. When RT is close to $200 \mathrm{~ms}$, the correlation coefficients decrease sharply. The correlation coefficients of TRS are larger than that of DSB except of individual cases. For TRS, the max and min of correlation coefficients are 0.6440 and 0.5016 respectively even SNR is $-15 \mathrm{~dB}$. So TRS outperforms DSB in reverberation environment.

\section{Effect of noise}

In order to show the anti-noise ability of TRS, this section discusses the effect of noise on separation. Under basic simulation condition and RT $=156 \mathrm{~ms}$, when white noise with different SNR is added into the array signals, the correlation coefficients between the separation signals and original signals for two methods are illustrated in Fig.3. 

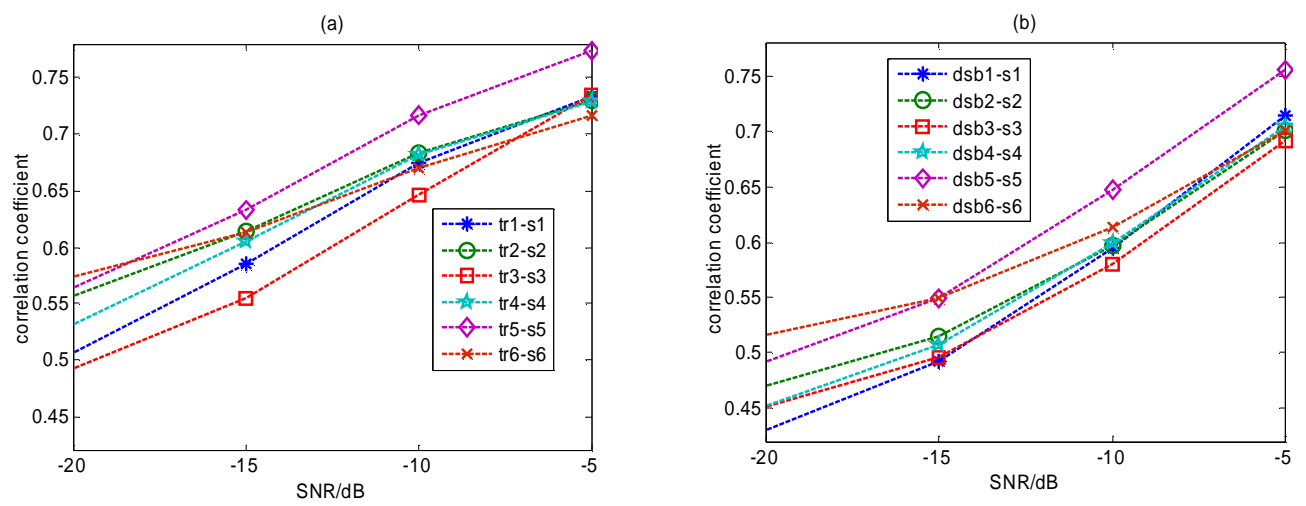

Fig.3 (a) Correlation coefficients between tr1-tr6 and s1-s6 with different SNR and RT=156 ms. (b) Correlation coefficients between dsb1-dsb6 and s1-s6 with different SNR and RT=156 ms.

Results depicted in Fig. 3 show that for TRS and DSB, the correlation coefficients increase with the SNR increasing. However, the correlation coefficients between tr1-tr6 and s1-s6 are larger than those between dsb1-dsb6 and s1-s6. The lower the SNR is, the bigger the differences between the correlation coefficients of two methods are. For TRS, the max and min of correlation coefficients are 0.7733 (tr5-s5, SNR $=-5 \mathrm{~dB})$ and $0.4933(\operatorname{tr} 3-\mathrm{s} 3, \mathrm{SNR}=-20 \mathrm{~dB})$ respectively even RT is 156 ms. TRS has better anti-noise ability than DSB.

\section{Conclusion}

In the present paper, the method of TRS was proposed and the theory and simulation experiments were presented. In a 3D rectangular room model with reverberation, six scattered sound sources were separated based on TRS with scattered sixteen mics. We discussed the effects of reverberation and noise on separation. We also compared the presented method of TRS with DSB. Simulation results show that multi-source can be separated by TRS; under reverberation and noise, TRS can achieve good separation effect; TRS has better separation effect than DSB under the same simulation condition.

\section{Acknowledgements}

The work was supported by National Natural Science Foundation of China (61471310).

\section{References}

[1] Yun Li, K.C.Ho, Mihail Popescu, Efficient source separation algorithms for acoustic fall detection using a Microsoft Kinect, IEEE Trans Biomed Eng 99 (2013) 1-9.

[2] Hong-Guang Ma, Qin-Bo Jiang, Zhi-Qiang Liu, Gang Liu, Zhi-Yuan Ma, A novel blind source separation method for single-channel signal, Signal Proc 90 (2010) 3232-3241.

[3] M.El Rhabia, H.Fenniri, A.Keziou, E.Moreau, A robust algorithm for convolutive blind source separation in presence of noise, Signal Proc 93 (2013) 818-827.

[4] A.S.Bregman, Auditory scene analysis, MIT Press, Cambridge, 1990.

[5] Jacob Benesty, Jingdong Chen, Yiteng Huang, Microphone Array Signal Processing, Springer Topics in Signal Processing, vol. 1, Berlin, Heidelberg, 2008.

[6] Fu Yongqing, Jiang Yulei, Liu Zhanya, Near-field source localization method and application using the time reversal mirror technique, J Electron (China) 28 (2011) 531-539.

[7] G.Ribay, J.De Rosny, M.Fink, Time Reversal of noise sources in a reverberation room, J Acoust Soc Am 
117 (2005) 2866-2872.

[8] J.de Rosnv, M.Fink, Overcoming the diffraction limit in wave physics using a time-reversal mirror and a novel acoustic sink, Phys Rev Lett 89 (2002) 1-4.

[9] Mathias Fink, Claire Prada, Acoustic time-reversal mirrors, Inverse Probl 17 (2001) 1-38.

[10] Mathias Fink, Time reversal of ultrasonic fields-part I: basic principles, IEEE Trans Ultrason Ferroelectr Freq Control 39 (1992) 555-567.

[11] J.M.Parot, Localizing impulse sources in an open space by Time Reversal with very few transducers, Appl Acoust 69 (2008) 311-324.

[12] Jont B.Allen, David A.Berkley, Image method for efficiently simulating small-room acoustics, J Acoust Soc Am 65 (1979) 943-951. 